\title{
THE GALACTIC DISTRIBUTION OF ELECTRON DENSITY MICROSTRUCTURE INFERRED FROM RADIO SCATTERING OBSERVATIONS *
}

\author{
JAMES CORDES \\ Astronomy Department, Cornell University
}

\begin{abstract}
I first review the observables and optics of interstellar seeing associated with radio wave scattering in the interstellar medium. I then describe the Galactic distribution of electron density and its fluctuations, as inferred from a number of observables, including angular and pulse broadening, diffractive scintillations, and dispersion measures. Propects for improving the Galactic model are outlined.
\end{abstract}

Keywords: interstellar medium, electron density, turbulence

\section{The Optics of ISS}

Interstellar scattering and scintillation (ISS) observables derive from the basic astrophysical configuration of radio source, scattering material and observer shown in Figure 1. The figure shows the density variations confined to a slab. In the Galaxy, turbulence is ubiquitous in the ionized component of the ISM, though highly inhomogeneous. The geometry changes on time scales dictated by the velocities of the source, medium and observer. For pulsars, the source velocity typically dominates while, for extragalactic sources, the velocities of the observer and ISM (including differential Galactic rotation) dominate. Viewed from a sufficiently large distance, the wavefront distortions are manifested as a rich variety of phenomena. Figure 2 shows examples of dispersive propagation, pulse broadening, and DISS. Rickett (1990) reviews many of the phenomena and issues. Here we describe briefly some of them:

Dispersive arrival times: Pulsar pulses arrive differentially with frequency according to delays, $\Delta t(v) \propto v^{-2} \mathrm{DM}$, where the dispersion measure is the integral DM $=\int_{0}^{D} d s n_{e}(s)$.

Angular broadening ('seeing'): Compact radio sources of many kinds, Galactic and extragalactic, show angular broadening by amounts ranging from $\lesssim 1$ mas to $1 \operatorname{arcsec}$ at $1 \mathrm{GHz}$, depending on the distance and direction. The angular smearing scales approximately as $v^{-2}$.

* Work supported by NSF Grant 9819931 . 
Time of arrival fluctuations: Changes in geometry induce DM variations. Also, variable scattering causes variable arrival times.

Pulse broadening: Multipath propagation causes a multiplicity of arrival times, usually seen as an exponential-like 'tail' to pulses from pulsars.

Diffractive intensity scintillations (DISS): Intensity variations in time and frequency on times scales $\sim 100 \mathrm{sec}$ and frequency scales $\sim 1 \mathrm{MHz}$. These scales are very highly dependent on frequency, direction and source distance and velocity. These variations result from diffraction of radiation into an angle $\theta_{d} \sim \lambda / \ell_{d}$, where $\ell_{d}$ is the diffraction scale, the transverse length on which the phase perturbation from $\delta n_{e}$ is one radian.

Refractive intensity scintillation (RISS): Intensity variations from large scale focusing and defocusing of the radiation. The refraction scale is $\ell_{r} \sim D \theta_{d}$,

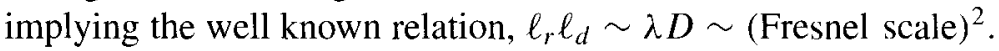

Spectral Broadening: Broadening of narrow spectral lines from a combination of scattering and time-variable geometry. The effect is very small in the ISM $(\lesssim 1 \mathrm{~Hz})$ but has been measured from spacecraft viewed through the interplanetary medium.

Superresolution phenomena: The optical/atmospheric 'Stars twinkle but planets do not' translates into 'Pulsars twinkle, AGNs do not,' at least for DISS. The (angular) isoplanatic scale for DISS is typically $\sim \ell_{d} / D \lesssim 10^{-6}$ arc sec at $1 \mathrm{GHz}$, or about $\times 10^{3}$ smaller than an AGN and $\times 10$ larger than a typical pulsar magnetosphere. Thus, we expect pulsars to show fully modulated scintillations in the strong scattering regime, whereas AGNs will not show DISS, typically. RISS is more forgiving of a large source size because it is caused by much larger turbulence scales than is DISS, with a critical angle $\sim \ell_{r} / D \sim \theta_{d}$. Thus, AGNs and masers, as well as pulsars, show RISS.

\section{Measure for Measure}

The dispersion measure DM was defined above as the integral of the electron density along a line of sight. The emission measure is the integral of the square of the electron density, $\mathrm{EM}=\int_{0}^{D} d s n_{e}^{2}(s)$.

Scattering measurements yield estimates of the scattering measure $\mathrm{SM}=\int_{0}^{D} d s$ $C_{n}^{2}(s)$, where $C_{n}^{2}$ is the spectral coefficient of the wavenumber spectrum of $\delta n_{\iota}$, assumed to be a power-law in wavenumber $q$ :

$$
P_{\delta n_{e}}(q)=C_{n}^{2} q^{-\beta}, \quad \frac{2 \pi}{\ell_{0}} \leq q \leq \frac{2 \pi}{\ell_{1}},
$$




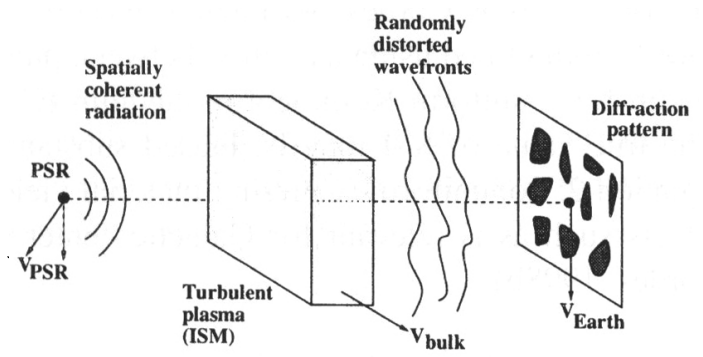

Figure /. The geometry of scattering and scintillation. A pulsar emits radiation with high spatial coherence. Wavefronts are perturbed by electron density variations $\delta n_{e}$ in the interstellar medium (ISM) which cause refractive index fluctuations, $\delta n_{r} \approx-2 \pi r_{e} \lambda^{2} \delta n_{e}$, where $r_{e}$ is the classical electron radius and the expression holds for frequencies much larger than the plasma frequency, $v_{p} \approx 2 \mathrm{kHz}\left(n_{e} / 0.03 \mathrm{~cm}^{-3}\right)^{1 / 2}$.
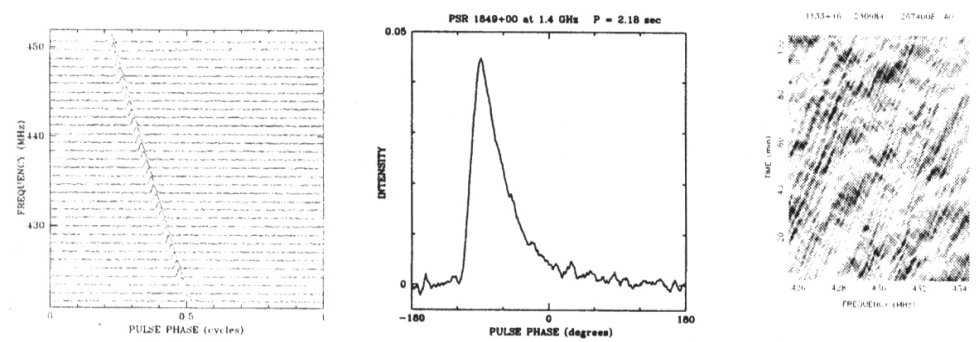

Figure 2. Left: Dispersive arrival times of PSR B1749-28 over a $32 \mathrm{MHz}$ bandwidth at 0.4 GHz. Middle: Pulse broadening of PSR B1849+00 at $1.4 \mathrm{GHz}$. Right: Dynamic spectrum of PSR B $1133+16$.

where $\ell_{1}, \ell_{0}$ are the inner and outer scales of the turbulence and the spectrum vanishes outside the specified interval. Equation 1 explicitly assumes isotropic irregularities. Evidence for anisotropies exists but it is not clear on what length scale these are present (see below). For the remainder of our discussion, we assume $\beta=11 / 3$ when evaluating expressions for the scattering measure.

From observations of the seeing disk size, the scintillation bandwidth and the pulse broadening time, SM may be estimated (with units $\mathrm{kpc} \mathrm{m}^{-20 / 3}$ ):

$$
S M= \begin{cases}\left(\theta_{d} / 128 \mathrm{mas}\right)^{5 / 3} v^{11 / 3} & \text { Extragalactic angular broadening } \\ \left(\theta_{d} / 71 \mathrm{mas}\right)^{5 / 3} v^{11 / 3} & \text { Galactic angular broadening } \\ 0.23\left(\Delta v_{d} D\right)^{-5 / 6} v^{11 / 3} & \text { DISS bandwidth } \\ 0.92\left(\tau_{d} / D\right)^{5 / 6} v^{11 / 3} & \text { Pulse broadening }\end{cases}
$$

where $\theta_{d}$ is the seeing disk size (milliarcsec), $\Delta v_{d}$ is the scintillation bandwidth $(\mathrm{kHz}$; calculated as the half-width at half maximum of the intensity correlation function), and $\tau_{d}$ is the pulse broadening time at $1 / e$ (milliseconds). The numerical values in Equations 2 assume a uniform medium for Galactic sources and plane wave incidence on the Galaxy's ISM for extragalactic sources. These expressions 
are consistent with those given by Cordes and Lazio (1991) but with the scintillation bandwidth expression modified using a relation between pulse broadening and scintillation bandwidth for a uniform Kolmogorov medium (Cordes and Rickett, 1998). Note that the true value of SM may be biased substantially from values estimated using Equation 2: nonuniform scattering material yields strong geometrical leveraging effects such as is relevant for Galactic center scattering (Lazio, 1997; Lazio and Cordes, 1998b).

\section{Wavenumber Spectrum of $\delta n_{e}$}

The wavenumber spectrum is well studied but there is contradictory information about its shape and extent in wavenumber. The spectrum is clearly not dominated by a single scale; evidence for a broad spectrum comes from scaling laws of propagation parameters with various quantities, such as interferometer baseline, frequency, and time. Y. Gupta (these proceedings) describes these constraints. A synthesis of measurements into a composite wavenumber spectrum is given by Armstrong, Rickett and Spangler (1995) and builds upon early work by Lee and Jokipii (1976).

If the spectrum is of the form of Equation 1 and if isotropic 3D turbulence is assumed, $\beta$ ranges between about 3.5 and 4 , including the 'Kolmogorov' value of $11 / 3$. The range of scales for which a power law is consistent is roughly from $\sim 10^{4} \mathrm{~km}$ to $\sim 10 \mathrm{AU}$, though the range varies considerably from object to object. For some very highly scattered objects, observations probe scales $\lesssim 10^{3} \mathrm{~km}$ and indicate the existence of an 'inner' scale. Weak constraints on the outer scale derive from a comparison of the scattering measure and the emission measure, yielding $\ell_{0} \sim 0.01 \mathrm{pc}$ for heavily scattered sources and $\ell_{0} \lesssim 100 \mathrm{pc}$ for weakly scattered sources.

There is substantial evidence that the turbulence is anisotropic, at least for some directions (e.g. Frail et al., 1994). Heavily scattered lines of sight toward Cygnus X-3, NGC6334B, Sgr A*, OH/IR masers in the Galactic center, and other Cygnus sources show seeing disks that are elliptical, with axial ratios as large as 4 . Because heavy scattering is probably concentrated to a small fraction of the total distance to the source, a likely interpretation is that the turbulence is two dimensional with an axis defined by the local magnetic field. One might expect the anisotropy to disappear in observations of sources where scattering occurs everywhere, and about equally, along the line of sight. Thus, while anisotropy is clearly established in some directions, there is a selection effect associated with those directions. It is therefore premature to conclude that anisotropy is associated with all scattering material.

The spectrum appears to be close to the Kolmogorov form $(\beta=11 / 3$ for isotropic turbulence, $8 / 3$ for $2 \mathrm{D}$ turbulence) for some directions at some epochs. However, there are large episodic changes in propagation effects, including DM 
fluctuations, multiple imaging of pulsars (as inferred from oscillations in dynamic spectra), and events in the light curves of AGNs ('extreme scattering events,' ESEs; see M. Walker, these proceedings) that suggest the existence of deterministic structures that superpose with a Kolmogorov process. Alternatively, there could be purely power-law processes but with widely different inner scales that superpose and cause the variable phenomena.

\section{Galactic Distribution}

The distribution of dispersing and scattering gas in the Galaxy (i.e. the nonrelativistic plasma) is complex, owing to the rich phase structure of the ISM and dynamical behavior driven by Galactic rotation and by supernova blast waves.

The three measures, DM, EM and SM, along with auxiliary distance information (from HI absorption, parallaxes and associations with supernova remnants and globular clusters), may be used to model both the local mean electron density and the fluctuations responsible for scattering. Details are given in Cordes et al.(1991) and Taylor and Cordes (1993). If one assumes that ionized gas is distributed in small clouds with volume filling factor $f$ and with $\epsilon=\delta n_{e} / n_{e}$ inside clouds, we have the relationships,

$$
\begin{aligned}
\mathrm{EM} & =\zeta \bar{n}_{e} \mathrm{DM}+C_{\mathrm{SM}}{ }^{-1} \ell_{0}^{2 / 3} \mathrm{SM} \\
\frac{\mathrm{SM}}{\mathrm{DM}} & =C_{\mathrm{SM}}\left\langle n_{e}\right\rangle_{\text {l.o.s. }}\left[\frac{\zeta \epsilon^{2}}{f \ell_{0}^{2 / 3}}\right],
\end{aligned}
$$

where $\zeta$ is the ratio of mean-square to squared mean density as it varies from cloud to cloud; $C_{\mathrm{SM}}$ is a constant $\sim 1$; and $\left\langle n_{e}\right\rangle_{\text {l.o.s. }}$ is the line of sight averaged electron density. The relationship of EM to DM and SM is given in the first expression. The second expression indicates that SM/DM should be constant for a medium with homogeneous statistics. Empirically we find instead that the ratio varies by a factor of at least 20 in going from the solar neighborhood to lines of sight that probe the inner Galaxy, especially the molecular ring. Evidently, the properties of clouds or their sizes and separations change dramatically.

A model that incorporates many kinds of measurements is the electron density model (with fluctuations) of Taylor and Cordes (1993). It includes a tenuous thick disk component, a thin disk component, spiral arms, and a molecular ring. Spiral arms were put in by hand but with parameters fitted by chi-square. The amplitudes and scale lengths (radial and in the $z$ direction) for other components were also fitted for. The model is clearly deficient in many respects, especially for lines of sight to the Galactic center and toward the outer Galaxy. Lazio (1997) used VLA and VLBI observations to better constrain these components (Lazio and Cordes, 1998ab). There are also pulsars for which the model over or underpredicts the pulse broadening. A new version of the model is being worked on (Cordes and Lazio, 
in preparation) that includes these new features as well as new definitions of the spiral arms. In addition, the electron density model will improve substantially using measurements on new pulsars from the Parkes multibeam survey. A collaboration between J. Cordes, J. Lazio and the Parkes Multibeam team is planned.

Finally, the distribution of scattering material is highly constrained by using DISS observations along with proper motions of pulsars. Cordes and Rickett (1998) have outlined an iterative, hybrid scheme that exploits all the information in such measurements.

\section{Acknowledgements}

I thank S. Chatterjee and M. McLaughlin for help preparing the figures and, along with R. Bhat, P. Goldreich, J. Lazio, R. Manchester, M. Walker and B. Rickett, for helpful discussions.

\section{References}

Armstrong, J.W., Rickett, B.J. and Spangler, S.R.: 1995, Electron Density Power Spectrum in the Local Interstellar Medium, Astrophys. J. 443, 209-221.

Cordes, J.M. and Lazio, T.J.W.: 1991, Interstellar Scattering Effects on the Detection of Narrow-band Signals, Astrophys. J. 376, 123-134.

Cordes, J.M. and Rickett, B.J.: 1998, Diffractive Interstellar Scintillation Time Scales \& Velocities, Astrophys. J. 507, 846-860.

Cordes, J.M., Weisberg, J.M., Frail, D.A., Spangler, S.R. and Ryan, M.: 1991, The Galactic Distribution of Free Electrons, Nature 354, 121-124.

Frail, D.A. et al.: 1993, Anisotropic Scattering of OH/IR Stars Toward the Galactic Center, Astrophys. J. Lett. 427, L43-46.

Lazio, T.J.W.: 1997, Genetic Algorithms, Pulsar Planets and Ionized Interstellar Microturbulence, PhD Thesis, Cornell University.

Lazio, T.J.W. and Cordes, J.M.: 1998a, The Radial Extent and Warp of the lonized Galactic Disk. II,, Astrophys. J. 497, 238-252.

Lazio, T.J.W. and Cordes, J.M.: 1998b, Hyperstrong Radio-Wave Scattering in the Galactic Center. I., II, Astrophys. J. 505, 715-731.

Lee, L.C. and Jokipii, J.R.: 1976, The Irregularity Spectrum in Interstellar Space, Astrophys. J. 206, $735-743$.

Rickett, B.J.: 1990, Radio Propagation Through the Turbulent Interstellar Plasma, Annu. Rev. Astron. Astrophys. 28, 561-605.

Taylor, J.H. and Cordes, J.M.: 1993, Pulsar Distances and the Galactic Distribution of Frec Electrons, Astrophys. J. 411, 674-684. 